\title{
pharmaceutics
}

ISSN 1999-4923

www.mdpi.com/journal/pharmaceutics

Article

\section{Effects of Mitragynine and a Crude Alkaloid Extract Derived from Mitragyna speciosa Korth. on Permethrin Elimination in Rats}

\section{Kachamas Srichana $^{1}$, Benjamas Janchawee ${ }^{1,2, *}$, Sathaporn Prutipanlai ${ }^{1}$, Pritsana Raungrut ${ }^{3}$ and Niwat Keawpradub ${ }^{4}$}

1 Department of Pharmacology, Faculty of Science, Prince of Songkla University, Songkhla 90112 , Thailand; E-Mails: hungayonko_o@hotmail.com (K.S.); sataporn.p@psu.ac.th (S.P.)

2 Natural Product Research Center, Faculty of Science, Prince of Songkla University, Songkhla 90112, Thailand

3 Department of Biomedical Sciences, Faculty of Medicine, Prince of Songkla University, Songkhla 90112, Thailand; E-Mail: rpritsan@medicine.psu.ac.th

4 Department of Pharmacognosy and Pharmaceutical Botany, Faculty of Pharmaceutical Sciences, Prince of Songkla University, Songkhla 90112, Thailand; E-Mail: niwat.k@psu.ac.th

* Author to whom correspondence should be addressed; E-Mail: benjamas.j@psu.ac.th; Tel.: +66-74-288171; Fax: +66-74-446678.

Academic Editor: Afzal Mohammed

Received: 16 November 2014 / Accepted: 13 March 2015 /Published: 27 March 2015

\begin{abstract}
Detoxification and elimination of permethrin (PM) are mediated by hydrolysis via carboxylesterase (CES). Mitragyna speciosa (kratom) contains mitragynine (MG) and other bioactive alkaloids. Since PM and MG have the same catalytic site and M. speciosa is usually abused by adding other ingredients such as pyrethroid insecticides, the effects of MG and an alkaloid extract (AE) on the elimination of PM were investigated in rats. Rats were subjected to single and multiple pretreatment with $\mathrm{MG}$ and $\mathrm{AE}$ prior to receiving a single oral dose $(460 \mathrm{mg} / \mathrm{kg})$ of PM. Plasma concentrations of trans-PM and its metabolite phenoxybenzylalcohol (PBAlc) were measured. The elimination rate constant ( $\mathrm{kel}_{\mathrm{el}}$ ) and the elimination half-life ( $\left.t_{1 / 2} \mathrm{el}\right)$ of PM were determined, as well as the metabolic ratio (PMR). A single and multiple oral pretreatment with $\mathrm{MG}$ and $\mathrm{AE}$ altered the plasma concentrationtime courses of both trans-PM and PBAlc during 8-22 h, decreased the PMRs, delayed elimination of PM, but enhanced elimination of PBAlc. Results indicated that PM-MG or
\end{abstract}


AE toxicokinetic interactions might have resulted from the $\mathrm{MG}$ and $\mathrm{AE}$ interfering with PM hydrolysis. The results obtained in rats suggest that in humans using kratom cocktails containing PM, there might be an increased risk of PM toxicity due to inhibition of PM metabolism and elimination.

Keywords: kratom; pyrethroids; toxicokinetic interaction; metabolic ratio

\section{Introduction}

Mitragyna speciosa Korth. (Rubiaceae), known as "kratom" in Thai, is indigenous to Thailand and other countries in Southeast Asia [1]. In the past, leaves of M. speciosa were chewed as an opium substitute before it was banned [2]. Thai villagers in the South have used it for a long time as a traditional medicine to relieve tiredness and muscle fatigue, and to treat some common illnesses such as diarrhea, coughing, muscle pain, diabetes, and hypertension [3,4]. It is representative of a particular social and local culture in southern Thailand. However, it has negative health impacts such as withdrawal symptoms due to addiction [4]. Health benefits of M. speciosa are due to the stimulatory and opioid-like effects of its bioactive constituents, such as a major alkaloid mitragynine (MG; Figure 1) and a minor component 7-hydroxymitragynine [5,6]. Several studies have revealed a number of pharmacological effects of $M$. speciosa, such as antinociception, antidiarrhea, antiinflammation, inhibition of gastric acid secretion, and stimulation of glucose transport in muscle cells [5-10]. In addition, it has been reported that it is used to treat opioid withdrawal [11].
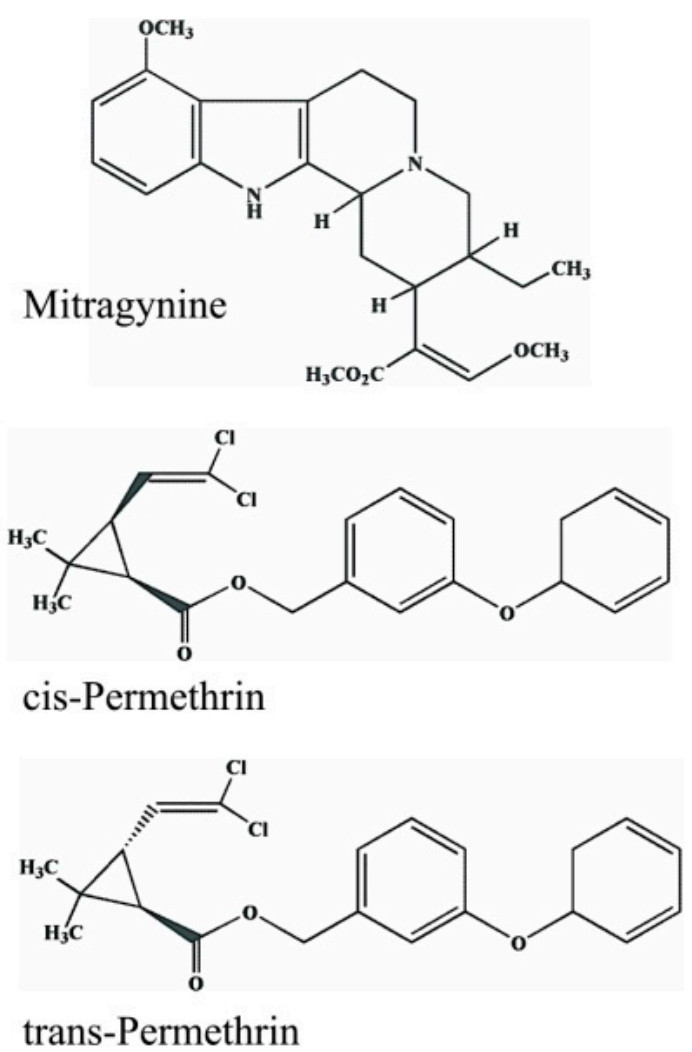

Figure 1. Chemical structures of mitragynine and permethrin (cis- and trans-isomers). 
Misuse of $M$. speciosa is especially found in Southern Thailand among adolescents. Users consume it in a form of cocktail, for example the " $4 \times 100$ " formulae containing boiled leaf extract, cola beverages, and codeine- or diphenhydramine-containing cough syrup as the basic ingredients. Other components are mosquito coils, anxiolytic drugs, abuse drugs such as methadone and methamphetamine, herbicide, or the powder peeled from the inside of fluorescent light bulbs. A toxicological analysis of the blood and urine to determine the cause of death of one young man revealed the presence of several substances found in " $4 \times 100$ " such as MG, alprazolam, nortriptyline, tramadol, and methamphetamine. Such a combination of multiple drugs can result in additive or synergistic adverse effects, especially depression of the central nervous system and the respiratory system, and that was probably the cause of his death [12].

A mosquito coil usually contains synthetic pyrethroid insecticides, substances structurally modified from pyrethrum extract. Common features of all pyrethroids include the presence of an acid moiety (central ester bond) and an alcohol moiety, and they are usually present as a stereoisomer. Pyrethroids are divided into two subclasses: type I (e.g., allethrin, permethrin (PM; Figure 1) and type II (e.g., deltamethrin, cypermethrin), based on the absence or presence, respectively, of a cyano group at the $\alpha$-carbon of the alcohol moiety [13]. The toxic effects of pyrethroids on insects and mammals are produced by disrupting the function of voltage-sensitive sodium channels in nerve cell membranes. In rodents, type I-pyrethroids produce the T (tremor) syndrome, while type II-congeners generate CS (choreoathetosis with salivation) syndrome [14]. Detoxification of pyrethroids such as permethrin (Figure 2) in mammals depends on its rapid hydrolysis by carboxylesterase (CES) to the constituent alcohol, which is further oxidized to an aldehyde, then to a carboxylic acid in the liver, prior to excretion into the urine and feces $[14,15]$.

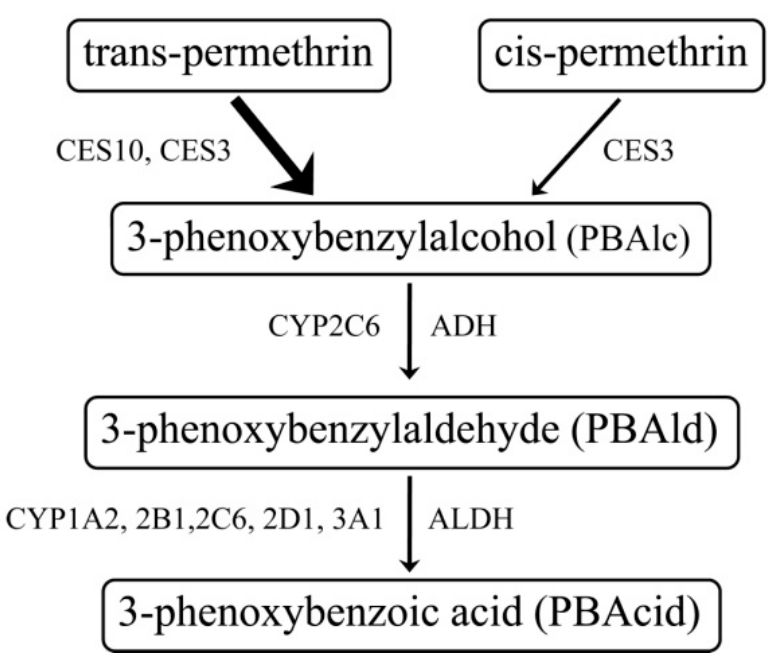

Figure 2. Scheme representing the metabolic pathway of permethrin in rat liver [16].

Investigation of the metabolites of $\mathrm{MG}$ in rats by liquid chromatographic mass spectrometry has indicated that MG was metabolized via hydrolysis of the methylester [17]. It may be hypothesized that MG affects the hydrolytic inactivation of pyrethroids, causing a delay in their elimination. That may increase the risk of toxicity of a synthetic pyrethroid in kratom cocktail users. A common synthetic pyrethroid used in mosquito coils is allethrin, yet PM is used since it is more photostable with comparative insecticidal activity. Since PM detoxification via hydrolysis occurs both in humans and 
rats, the present study was therefore meant to investigate any effects from MG and alkaloid extract (AE) derived from leaves of M. speciosa on the elimination of PM in rats.

\section{Experimental Section}

\subsection{Chemicals and Reagents}

Standard PM (purity 98\%; a mixture of cis- and trans-isomers; 26.4:71.7; PESTANAL ${ }^{\circledR}$ ) and phenoxybenzyl alcohol (PBAlc; purity 98\%) were from Sigma-Aldrich (Steinheim, Germany) and Aldrich (St. Louis, MO, USA), respectively. Methanol and acetonitrile (HPLC grade), and perchloric acid (AR grade) were from Mallinckrodt Baker Inc. (Phillipsburg, NJ, USA). Chloroform (AR grade) was from VWR International (Fontenay-sous-Bois, France). Acetic acid (AR grade) was from Merck (Darmstadt, Germany). Heparin (NUPARIN ${ }^{\circledR}$ ) was from Troikaa Pharmaceuticals (Gujarat, India).

\subsection{Plant Alkaloids}

Crude AE and MG were obtained from previous studies [18,19]. Leaves of $M$. speciosa were dried and extracted with methanol and chloroform with a $\mathrm{pH}$ modification to yield crude AE, which was further separated by using normal phase column chromatography. The pure compounds were identified by using MS (Thermofinnigan MAT 95 XL mass spectrometer: EIMS with direct insert probe; Thermo Finnigan MAT GmbH, Bremen, Germany), and ${ }^{1} \mathrm{H}-\mathrm{NMR}$ and ${ }^{13} \mathrm{C}-\mathrm{NMR}$ (Varian Unity Inova 500 NMR spectrometer, McKinley Scientific, Sparta, NJ, USA) spectra. Procedures for the extraction and isolation of crude alkaloids and MG have been described in detail elsewhere [18,20,21].

\subsection{Animals}

The experimental protocol was approved by the Animal Ethic Committee for Experimental Animals, Prince of Songkla University (Ref. 13/2012, approved 29 March 2012). Animal handling was in accordance with the guidelines of the National Research Council of Thailand based on the International Guiding Principles for Biomedical Research Involving Animals [22]. The experimental work also followed the ARRIVE guidelines [23]. Adult male Wistar rats (Mlac:WR; 200-220g) were obtained from the Southern Laboratory Animals Facility, Prince of Songkla University, Songkhla, Thailand. They were housed in a controlled environment ( ca. $23{ }^{\circ} \mathrm{C}, 12 \mathrm{~h}$ dark/light cycles) with food and water ad libitum. They were fasted overnight with free access to water prior to treatment.

\subsection{Experimental Design}

Four groups of animals ( $n=6$ each) were assigned different pretreatments; gr. I, MG $40 \mathrm{mg} / \mathrm{kg}$, per os (p.o.), once; gr. II, AE $100 \mathrm{mg} / \mathrm{kg}$, p.o. once; gr. III, MG $40 \mathrm{mg} / \mathrm{kg}$, p.o., 4 days; gr. IV, AE 100 $\mathrm{mg} / \mathrm{kg}$, p.o., 4 days. MG and AE were prepared by dissolving in $20 \%$ tween 20 . The animals of each group were subjected to two phases of the experiment. In phase I, they were given a single oral dose of $460 \mathrm{mg} / \mathrm{kg}$ of PM prepared in corn oil. In phase II, the same animal was pretreated with either MG, or $\mathrm{AE}, 2 \mathrm{~h}$ before receiving the same dose of PM. The wash out period was 5 days. 
Blood samples were periodically collected by tail clipping without anesthesia at $0,8,14,18$ and $22 \mathrm{~h}$ after PM administration in phase I and phase II. One-half a milliliter of blood sample was placed in an ice-chilled microcentrifuge tube containing $50 \mu \mathrm{L}$ of heparin $(60 \mathrm{unit} / \mathrm{mL})$ and $2.5 \mu \mathrm{L}$ of perchloric acid. After thoroughly mixing, it was centrifuged at $1600 \times g$ at $4{ }^{\circ} \mathrm{C}$ for $15 \mathrm{~min}$. Plasma was separated and kept at $-20^{\circ} \mathrm{C}$ prior to analysis.

\subsection{Determination of Permethrin (PM) and Phenoxybenzylalcohol (PBAlc) in Plasma}

\subsubsection{Preparation of Standard Solutions}

An individual stock solution of $10 \mathrm{mg} / \mathrm{mL}$ of PM and PBAlc was prepared by dissolving with acetonitrile. Working standard solutions of PM and PBAlc were prepared as a mixture by diluting the stock solution with acetonitrile to different concentrations that ranged from 0.5 to $1024 \mu \mathrm{g} / \mathrm{mL}$. Standard solutions for calibration at concentrations of $0.1,0.2,0.4,0.8,1.6,3.2,6.4,51.2,102.4$, and $204.8 \mu \mathrm{g} / \mathrm{mL}$ were prepared by adding the appropriate concentrations of the working standard solutions to plasma blank samples (final volume $200 \mu \mathrm{L}$ ).

\subsubsection{Sample Extraction}

Plasma samples were extracted using the solid phase extraction (SPE) method with some modifications [24]. Samples $(200 \mu \mathrm{L})$ were thoroughly mixed by vortex with $60 \mu \mathrm{L}$ of $1 \mathrm{~N}$ acetic acid. The VertiPak ${ }^{\mathrm{TM}} \mathrm{C}_{18}$-Tubes $(200 \mathrm{mg} / 3 \mathrm{~mL}$, Vertical Chromatography, Nonthaburi, Thailand) cartridges were preconditioned with $3 \mathrm{~mL}$ of chloroform, methanol, methanol-water $(50 \%, v / v)$, and water. The cartridges were washed with $3 \mathrm{~mL}$ of methanol-water $(10 \%, v / v)$, and eluted with $3 \mathrm{~mL}$ of chloroform. The eluate was evaporated to dryness with a stream of nitrogen gas at room temperature. The residue was reconstituted with $200 \mu \mathrm{L}$ of acetonitrile and a $20 \mu \mathrm{L}$ aliquot was injected into the HPLC system.

\subsubsection{Chromatographic Instruments and Condition}

High performance liquid chromatography (HPLC) was performed using a Waters 2695 Separation Module connected with a Waters 5487 Dual $\lambda$ Absorbance detector (Milford, MA, USA). Chromatographic data were processed using the Empower ${ }^{\mathrm{TM}}$ Software System (Milford, MA, USA).

PM and PBAlc were analyzed using the reversed phase HPLC method modified from the previous study [25]. The Fortis ${ }^{\mathrm{TM}} \mathrm{C}_{18}$ column $(150 \times 4.6 \mathrm{~mm}$ i.d., $5 \mu \mathrm{m}$ particle size $)$ connected to a Sunfire ${ }^{\mathrm{TM}}$ $\mathrm{C}_{18}$ guard column $(20 \times 4.6 \mathrm{~mm}$ i.d., $5 \mu \mathrm{m}$ particle size $)$ was used for separation. The freshly prepared mobile phase was water and acetonitrile. Elution was performed using the gradient program starting at $65 \%$ acetonitrile for $5 \mathrm{~min}$, then increased to $100 \%$ acetonitrile from 5 to $15 \mathrm{~min}$, and decreased to $65 \%$ acetonitrile from 15 to $20 \mathrm{~min}$, with a post run of $7 \mathrm{~min}$ to equilibrate the column between injections. The flow rate of the eluent was constant at $1.4 \mathrm{~mL} / \mathrm{min}$. The column temperature was maintained at $25 \pm 2^{\circ} \mathrm{C}$. The injection volume was $20 \mu \mathrm{L}$. The analytes were detected at a wavelength of $230 \mathrm{~nm}$. 


\subsection{Method Validation}

The method of analysis was validated in accordance with the USFDA Guidance [26] in terms of linearity, precision, accuracy, recovery, and lower limit of quantification (LLOQ).

Linearity was determined by preparing standard plasma samples with different concentrations of PM $(0.1,0.2,0.4,0.8,1.6$, and $3.2 \mu \mathrm{g} / \mathrm{mL} ; n=4)$ and PBAlc $(0.1,0.8,6.4,51.2,102.4$, and $204.8 \mu \mathrm{g} / \mathrm{mL}$; $n=4)$. The calibration curves were constructed by plotting the peak area of the analyte $(y)$ against its concentrations $(x)$. Regression analysis for each calibration curve was performed to obtain the calibration equation and correlation coefficient $(r)$.

Precision was evaluated by using four quality control (QC) samples of PM $(0.1,0.2,0.8$, and $3.2 \mu \mathrm{g} / \mathrm{mL})$ and PBAlc $(0.1,0.8,51.2$, and $204.8 \mu \mathrm{g} / \mathrm{mL})$ that were prepared by adding different concentrations of standard solutions into the plasma blank samples. Intra-day precision was determined by assaying five samples of each concentration during the same day while the inter-day precision was determined by assaying the samples daily for five consecutive days. The precision was expressed as the relative standard deviation (RSD) and was calculated using the formula: $R S D(\%)=\frac{\text { standard deviation }}{\text { mean }} \times 100$. The level of acceptance was within 15\%RSD except at the LLOQ where 20\%RSD is acceptable.

The same concentration of QC samples was used for determining the accuracy. Intra-day accuracy was determined by analyzing five samples of each concentration on the same day while the inter-day accuracy was determined by assaying for five consecutive days. The accuracy was expressed as the deviation (DEV) and was calculated using the formula: $D E V(\%)=$ $\frac{\text { measured concentration-nominal concentration }}{\text { nominal concentration }} \times 100$. It was acceptable when the DEV was within $\pm 15 \%$ or $\pm 20 \%$ at the LLOQ.

Recovery by the extraction was determined at concentrations of $0.1,0.2,0.8$, and $3.2 \mu \mathrm{g} / \mathrm{mL}$ for PM and $0.1,0.8,51.2$, and $204.8 \mu \mathrm{g} / \mathrm{mL}$ for PBAlc $(n=5)$. The recovery was calculated using the expression: Recovery $(\%)=\frac{\text { response after extraction }}{\text { response after direct injection }} \times 100$.

The LLOQs of PM and PBAlc were defined as the lowest concentration on the calibration curve that could be determined with a signal-to-noise ratio $(S / N)$ of 5 .

Validation results (Table 1) showed that calibration curves of PM and PBAlc were linear with good correlation coefficient $(r \geq 0.9999)$. The method was shown to measure both trans-PM and PBAlc precisely and accurately. Both intra- and inter-day precisions were within the level of acceptance, i.e., $\pm 15 \%$ RSD and $\pm 20 \%$ RSD for the concentration at LLOQ [26]. PM and PBAlc were almost completely recovered from plasma, i.e., $\geq 80 \%$ for trans-PM and $\geq 91 \%$ for PBAlc. The LLOQs for trans-PM and PBAlc were $0.1 \mu \mathrm{g} / \mathrm{mL}$. 
Table 1. Method validation for analysis of trans-permethrin (PM) and phenoxybenzylalcohol (PBAlc) in rat plasma.

\begin{tabular}{cccc}
\hline \multirow{2}{*}{ Validation parameter } & \multicolumn{2}{c}{ Analyte } \\
& & trans-PM & PBAlc \\
\hline Range $(\mu \mathrm{g} / \mathrm{mL})$ & & $0.1-3.2$ & $0.1-208.4$ \\
Linearity & Slope & $22491 \pm 374.08$ & $34378 \pm 400.79$ \\
& $y$-Intercept & $(-) 239.08 \pm 107.93$ & $806.31 \pm 419.26$ \\
& $r$ & 0.9999 & 1.0000 \\
Precision $(\% \mathrm{RSD})$ & Intra-day & $2.24-7.63$ & $1.18-3.66$ \\
& Inter-day & $3.12-6.75$ & $2.02-3.46$ \\
Accuracy $(\% \mathrm{DEV})$ & Intra-day & $(-) 2.37-(+) 10.92$ & $(-) 12.89-(+) 1.79$ \\
& Inter-day & $(-) 7.85-(+) 3.44$ & $(-) 11.91-(-) 0.21$ \\
Recovery $(\%)$ & & $80.43-82.34$ & $91.53-95.07$ \\
LLOQ $(\mu \mathrm{g} / \mathrm{mL})$ & & 0.1 & 0.1 \\
\hline
\end{tabular}

\subsection{Data Analysis}

Semi-log plasma concentrations of trans-PM and PBAlc against time were plotted. The elimination rate constant $(\mathrm{kel})$ was determined from the slope of the terminal phase $\left(T_{14}-T_{22}\right)$ of the curve following the equation: $\mathrm{k}_{\mathrm{el}}=\frac{- \text { slope }}{2.303}$. The elimination half-life $\left(t_{1 / 2} \mathrm{el}\right)$ was estimated according to the equation: $t_{1 / 2 \mathrm{el}}=\frac{0.693}{\mathrm{k}_{\mathrm{el}}}$.

The permethrin metabolic ratio (PMR) was calculated as the concentration ratio between PBAlc and trans-PM as follows: $P M R=\frac{[P B A l c]}{[\operatorname{trans}-P M]}$. The change in PMR was calculated using the expression: $\%$ Change in $P M R=\frac{\left(P M R_{\text {phase II }}-P M R_{\text {phase } I}\right)}{P M R_{\text {phase }}} \times 100$.

All data were expressed as a mean \pm SEM. The phase I (without pretreatment) and phase II (after pretreatment) data were obtained from the same animal and were compared using paired $t$-test. Data derived from different groups of pretreatment were analyzed using one-way analysis of variance (ANOVA) followed by post hoc least significant difference test. A significant difference was considered to be $p<0.05$. Statistical analyses were performed using SPSS Statistics Bass 17.0 for Windows EDU (SPSS Inc. Chicago, IL, USA).

\section{Results}

\subsection{Determination of PM and PBAlc in Plasma}

Chromatographic profiles (Figure 3) show that PBAlc, trans-PM, and cis-PM were well separated from plasma interferences with an elution time of approximately $17 \mathrm{~min}$. 

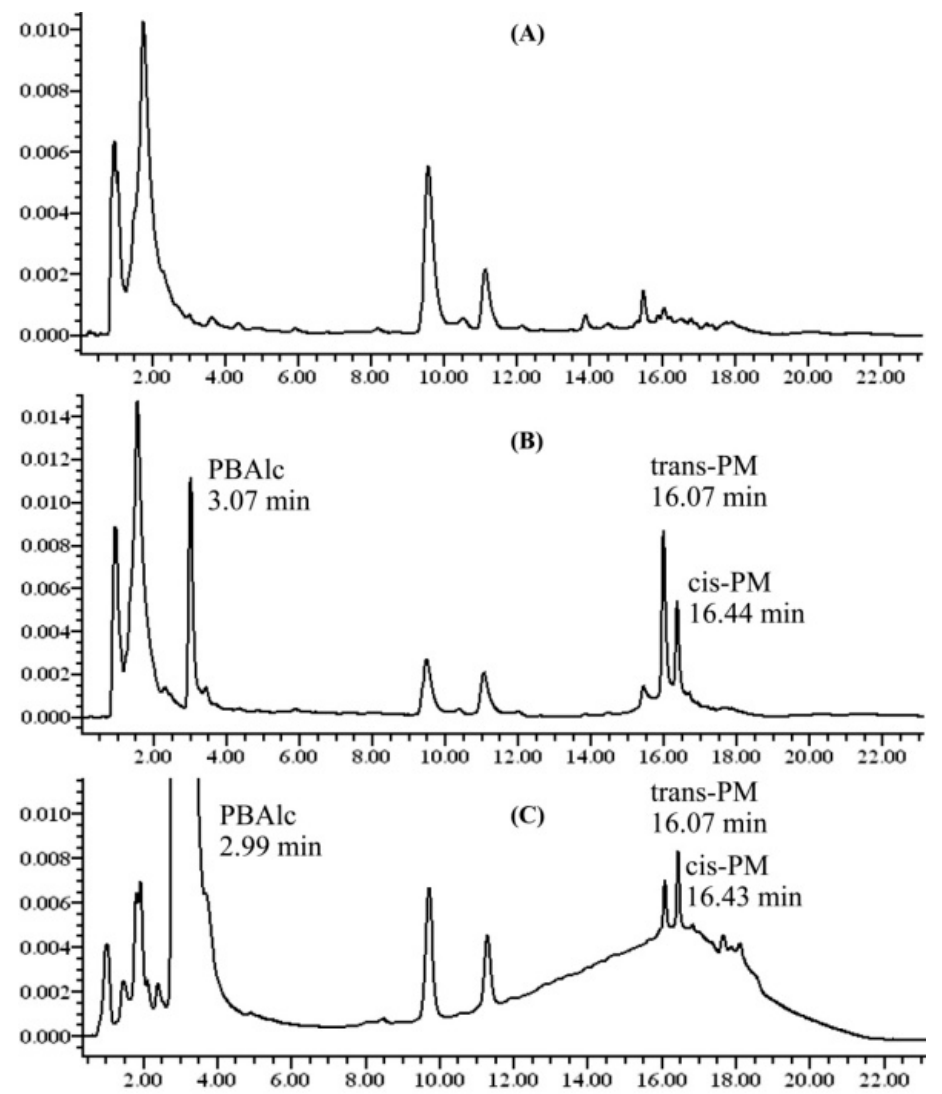

Figure 3. Representative chromatograms for the separation of PM and PBAlc in rat plasma; (A) blank plasma; (B) blank plasma spiked with standards of PM and PBAlc $(3.2 \mu \mathrm{g} / \mathrm{mL})$; (C) plasma sample at $8 \mathrm{~h}$ after dosing with PM $(460 \mu \mathrm{g} / \mathrm{mL}$, p.o.).

Plasma concentration-time profiles of trans-PM and PBAlc in rats pretreated with a single and multiple oral dose(s) of MG (40 mg/kg) and AE $(100 \mathrm{mg} / \mathrm{kg})$ are presented in Figure 4. PBAlc and trans-PM concentrations were measurable in the plasma during 8-22 $\mathrm{h}$ post dose and gradually declined over time. After $22 \mathrm{~h}$, the levels of trans-PM in the plasma of rats given a single dose and multiple dose pretreatment of MG were below the LLOQ.

The plasma concentrations of trans-PM after pretreatment with a single and multiple dose(s) of MG were significantly decreased at 8 and $14 \mathrm{~h}$ post dose compared with those without pretreatment (Figure 4A,B). However, the trans-PM concentrations at 18 and $22 \mathrm{~h}$ were relatively increased. Similar findings were noted for rats pretreated with a single and multiple dose(s) of AE (Figure 4C,D). In contrast, the plasma concentrations of PBAlc after pretreatment with a single and multiple dose(s) of MG and AE were significantly decreased at 8, 14, 18, and $22 \mathrm{~h}$.

The pharmacokinetic parameters that represent an elimination of trans-PM and PBAlc are presented in Table 2. Without pretreatment, the average values of the elimination rate constant (kel) of PM were similar and ranged from 0.059 to $0.062 \mathrm{~h}^{-1}$. The value of kel was significantly decreased after pretreatment with a single dose of $\mathrm{MG}$, i.e., from 0.061 to $0.035 \mathrm{~h}^{-1}$. Likewise, the kel for trans-PM was significantly reduced by administering multiple doses of $\mathrm{MG}$ and a single and multiple dose(s) of AE, i.e., from 0.062 to $0.033 \mathrm{~h}^{-1}$, from 0.060 to $0.031 \mathrm{~h}^{-1}$, and from 0.059 to $0.024 \mathrm{~h}^{-1}$, respectively. The decrease in the $\mathrm{k}_{\mathrm{el}}$ was the highest, i.e., about 2.5-fold, in the group that received AE multiple dose pretreatment. In contrast to PM, the elimination rate constants of PBAlc were significantly increased 
after pretreatment with a single dose of MG (from 0.035 to $0.075 \mathrm{~h}^{-1}$ ), multiple doses of MG (from 0.035 to $0.107 \mathrm{~h}^{-1}$ ), and multiple doses of $\mathrm{AE}$ (from 0.035 to $0.059 \mathrm{~h}^{-1}$ ). The highest increase (3.1 folds) was observed after pretreatment with multiple doses of MG.

(A)

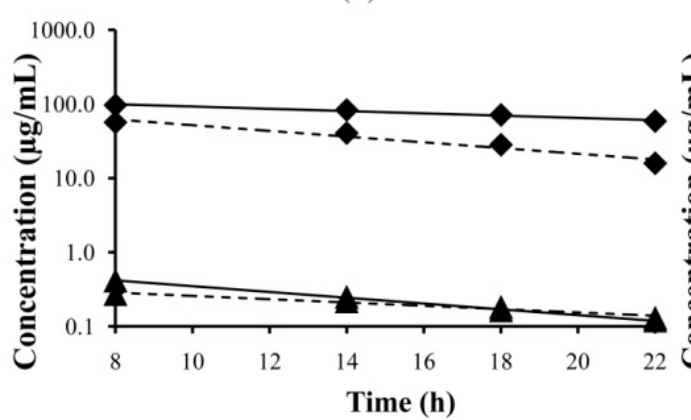

(C)

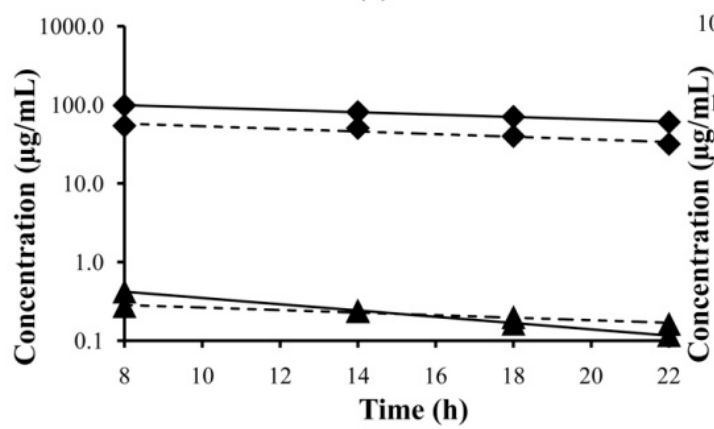

PBAlc, without pretreatment $\boldsymbol{\Delta}$ trans-PM, without pretreatment
(B)

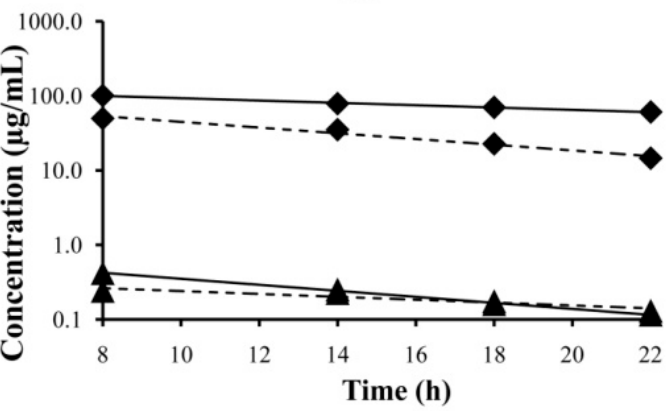

(D)

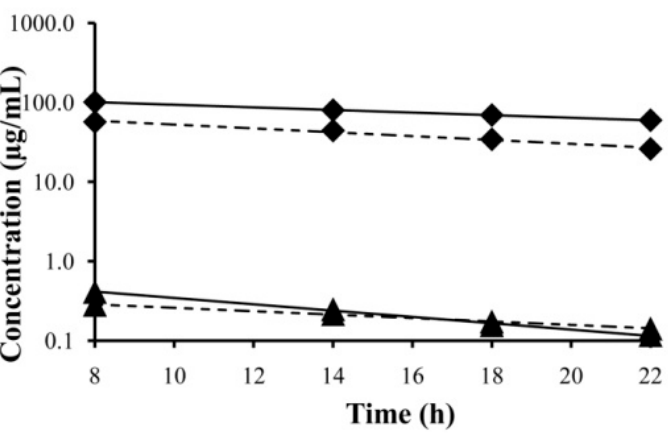

- - PBAlc, after pretreatment - - $\Delta$ trans-PM, after pretreatment

Figure 4. Semilog plasma concentration-time profiles of PBAlc and trans-PM during 8-22 $\mathrm{h}$ in rats receiving a single or multiple oral dose $(\mathrm{s})$ of $\mathrm{MG}(40 \mathrm{mg} / \mathrm{kg})$ and $\mathrm{AE}(100 \mathrm{mg} / \mathrm{kg})$; (A) MG single dose; (B) MG multiple dose; (C) AE single dose; (D) AE multiple doses.

Table 2. Elimination rate constants $\left(\mathrm{k}_{\mathrm{el}}\right)^{\dagger}$ and elimination half-lives $\left(t_{1 / 2} \mathrm{el}\right)^{\dagger}$ of $\mathrm{PM}$ and PBAlc in rats following a single and multiple dose(s) of MG and AE administration.

\begin{tabular}{|c|c|c|c|c|c|}
\hline \multirow{2}{*}{ Group } & \multirow{2}{*}{ Substance } & \multicolumn{2}{|l|}{$k_{\text {el }}\left(h^{-1}\right)$} & \multicolumn{2}{|l|}{$t_{1 / 2}$ el $(h)$} \\
\hline & & Without pretreatment & After pretreatment & Without pretreatment & After pretreatment \\
\hline I (MG single & $\mathrm{PM}$ & $0.061 \pm 0.001^{a}$ & $0.035 \pm 0.002^{* a}$ & $11.46 \pm 0.13$ & $19.98 \pm 1.17^{* a}$ \\
\hline dose) & PBAlc & $0.035 \pm 0.004$ & $0.075 \pm 0.007^{* A}$ & $20.75 \pm 2.13$ & $9.49 \pm 0.80 * \mathrm{~A}$ \\
\hline II (MG & $\mathrm{PM}$ & $0.062 \pm 0.001^{a}$ & $0.033 \pm 0.002 * \mathrm{a}$ & $11.19 \pm 0.12$ & $22.65 \pm 0.85^{* a}$ \\
\hline multiple dose) & PBAlc & $0.035 \pm 0.002$ & $0.107 \pm 0.008^{* \mathrm{~B}}$ & $20.46 \pm 0.82$ & $6.65 \pm 0.49 * \mathrm{~A}$ \\
\hline III (AE single & $\mathrm{PM}$ & $0.060 \pm 0.001^{\mathrm{a}}$ & $0.031 \pm 0.001^{* a}$ & $11.61 \pm 0.22$ & $22.69 \pm 0.68^{* a}$ \\
\hline dose) & PBAlc & $0.041 \pm 0.003$ & $0.042 \pm 0.005^{\mathrm{C}}$ & $17.41 \pm 1.34$ & $17.80 \pm 2.21^{\mathrm{B}}$ \\
\hline IV $(\mathrm{AE}$ & PM & $0.059 \pm 0.001^{\mathrm{a}}$ & $0.024 \pm 0.001 * \mathrm{~b}$ & $11.71 \pm 0.26$ & $29.08 \pm 1.47$ *b \\
\hline multiple dose) & PBAlc & $0.035 \pm 0.001$ & $0.059 \pm 0.002 * \mathrm{AC}$ & $20.06 \pm 0.80$ & $11.88 \pm 0.48 * \mathrm{AC}$ \\
\hline
\end{tabular}

${ }^{\dagger}$ Mean $\pm \operatorname{SEM}(n=6) ;{ }^{*} p<0.01$, compared with without pretreatment using paired $t$-test. Values within the same column that have different letter superscripts (lowercase for PM; uppercase for PBAlc) indicate a significant difference using ANOVA by post hoc least significant difference test, $p<0.01$. 
The baseline elimination half-lives of trans-PM in all groups of pretreatments ranged from 11.19 to $11.71 \mathrm{~h}$ (Table 2). Pretreatment with single and multiple doses of MG and AE significantly increased the elimination half-life of trans-PM, i.e., from 11.46 to $19.98 \mathrm{~h}, 11.19$ to $22.65 \mathrm{~h}, 11.61$ to $22.69 \mathrm{~h}$, and 11.71 to $29.08 \mathrm{~h}$, respectively. The increase in $t_{1 / 2}$ el was the greatest (2.5-fold) following administration of multiple doses of AE. The elimination half-lives of PBAlc were significantly decreased after pretreatment with a single dose of MG (from 20.75 to $9.49 \mathrm{~h}$ ), multiple doses of MG (from 20.46 to $6.65 \mathrm{~h}$ ), and multiple doses of AE (from 20.06 to $11.88 \mathrm{~h}$ ). The highest decrease (3.1 folds) was observed after pretreatment with multiple doses of MG.

Figure 5 represents the PMRs in rats without and after pretreatment with a single and multiple dose(s) of MG and AE. A single dose of MG caused a significant decrease in PMRs at 8, 14, 18, and $22 \mathrm{~h}$ after PM administration (Figure 5A). Similar results were observed in the pretreated rat groups receiving multiple doses of $\mathrm{MG}$ (Figure $5 \mathrm{~B}$ ), and single and multiple doses of AE (Figure $5 \mathrm{C}, \mathrm{D}$ ).
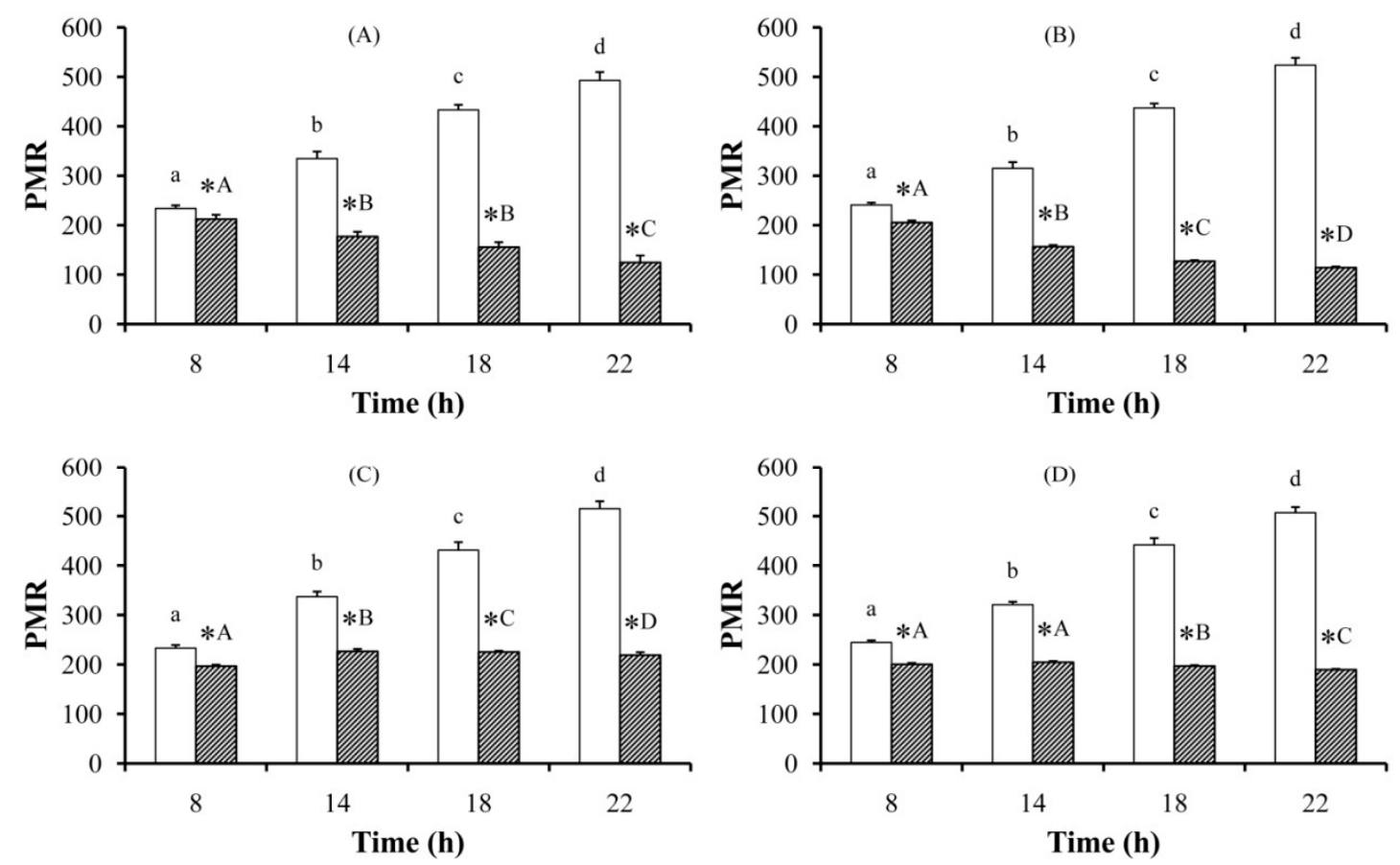

$\square$ Without pretreatment

After pretreatment

Figure 5. $\mathrm{PMR}^{\dagger}$ in rats receiving a single and multiple oral dose(s) of $\mathrm{MG}(40 \mathrm{mg} / \mathrm{kg})$ and AE (100 mg/kg); (A) MG single dose; (B) MG multiple doses; (C) AE single dose; (D) AE multiple doses. ${ }^{\dagger}$ Mean \pm SEM. Significance differences were considered at $p<0.05$. Asterisk represents significant difference when compared with without pretreatment at the same time using paired $t$-test. A different letter (a lowercase for without pretreatment; an uppercase for after pretreatment) indicates significant difference using ANOVA followed by least significant difference test.

The percent decrease in PMRs is illustrated in Figure 6. The percentage of the decrease was significantly increased with time for all groups of pretreatment.

At $8 \mathrm{~h}$, the percent reduction of PMRs tended to increase after pretreatment with multiple doses of MG or AE. It was the highest after the multiple-dose administration of AE. In contrast, at 14, 18, and 
$22 \mathrm{~h}$, the percent reduction of PMRs was significantly decreased after a single- and multiple-dose administration of AE compared to those after single- and multiple-dose administration of MG.

The percent reduction in PMRs tended to increase after pretreatment with multiple doses of either MG or AE, compared with those after a single dose. The reduction was significant at $18 \mathrm{~h}$ after PM administration.

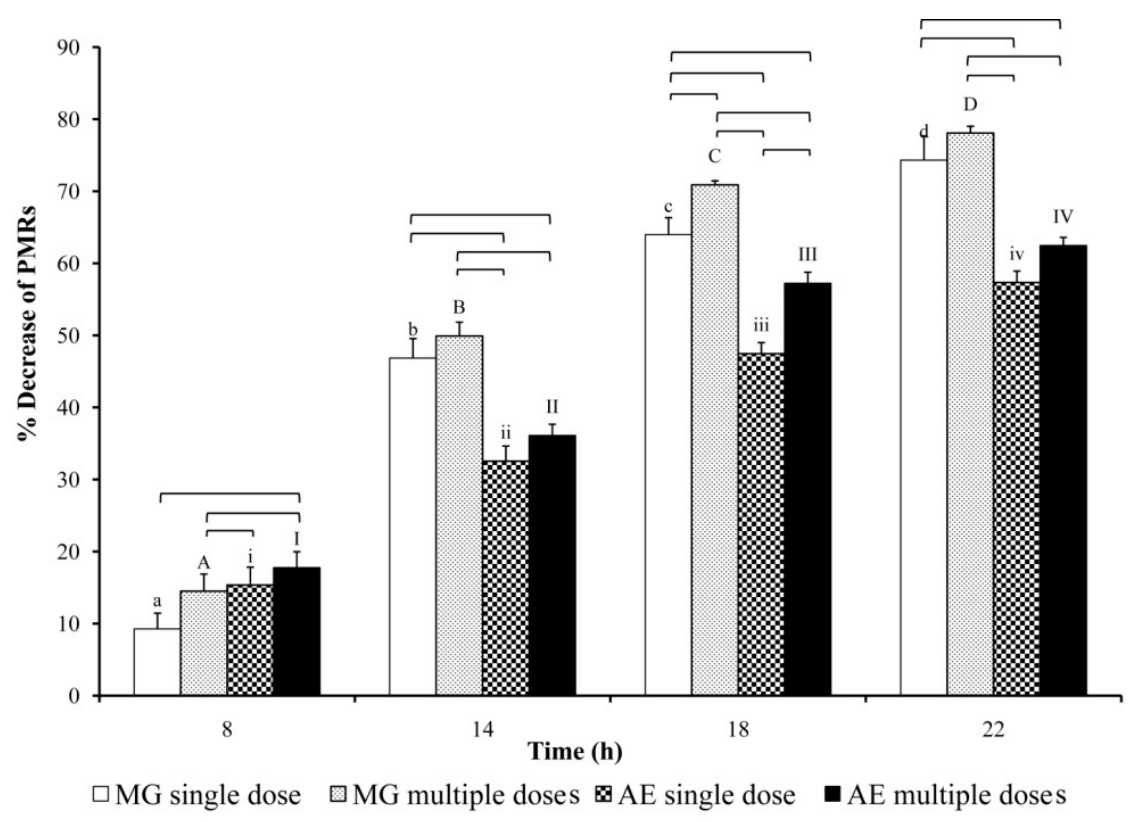

Figure 6. Percentage of the decrease in $\mathrm{PMRs}^{\dagger}$ in rats following a single and multiple oral administration of MG $(40 \mathrm{mg} / \mathrm{kg})$ and $\mathrm{AE}(100 \mathrm{mg} / \mathrm{kg}){ }^{\dagger}$ Mean $\pm \mathrm{SEM}$. Significance differences were considered at $p<0.05$ using ANOVA followed by least significant difference test. A different sign (a lowercase letter for MG single dose; an uppercase letter for MG multiple doses; a lower case roman number for AE single dose; an upper case roman number for AE multiple doses) indicates significant difference among the same treatment. The bracket indicates a pair of data having significant difference.

The results show that after pretreatment with MG or AE, the elimination of PM was slower, and the levels of the metabolite PBAlc was lower. These findings point to inhibited metabolism of PM.

\section{Discussion}

Hepatic metabolism of PM is responsible for its detoxification and elimination. The pathways and enzymes for PM metabolism have been documented in many in vitro studies. The study using human liver fractions showed that trans-PM was metabolized to PBAlc and phenoxybenzoic acid (PBAcid). To produce PBAlc, the ester bond of trans-PM was hydrolyzed by CES [27], a serine hydrolase catalyzing the hydrolysis of esters, amides, and thioesters [28]. PBAlc was then oxidized by alcohol dehydrogenase (ADH) to phenoxybenzaldehyde (PBAld) and subsequently to PBAcid by aldehyde dehydrogenase. cis-PM was not significantly metabolized. Both the hydrolysis of trans-PM and oxidation of PBAlc did not involve the cytochrome P450s. A study using rat microsomes showed that trans-PM was more effectively hydrolysed than cis-PM by liver microsomes and by the small intestinal microsomes of rats. The ES-3 and ES-10, isoforms of the CES1 family were responsible for 
the hydrolysis of trans-PM. Oxidation of the alcohol and aldehyde metabolites required cytochrome P450 [16]. Another study reported that metabolism of trans-PM in the rat and human liver microsomes were from the combination of both hydrolysis and oxidation while that of the cis-PM was solely the result of oxidation [29].

The MG and M. speciosa leaf extracts have been shown to interact with the drug-metabolizing enzymes. Based on human recombinant CYP enzymes, a methanolic extract of $M$. speciosa showed the most potent inhibition on CYP2D6 activity. The extract had a weak effect on CYP3A4 activity and a negligible effect on CYP2C9 activity [30]. It has been confirmed that MG had the strongest inhibitory activity on CYP2D6, while CYP2C9 and CYP3A4 are inhibited to a lesser extent [31]. An $M$. speciosa alkaloid extract had a moderate inhibitory effect on CYP1A2 [32], while MG exhibited an inductive effect on CYP1A2 [33]. In an in vitro study, the methanolic, aqueous, and total alkaloid extracts of $M$. speciosa leaves exhibited a concentration-dependent inhibition of glutathione $S$-transferase (GST), 61\%, 50\%, and 43\% inhibition, respectively, in rats, whereas the in vivo findings showed an enzyme induction after a 14-day treatment [34]. MG inhibited GST in a dose-dependent manner, but a high dose was required to induce the activity of $N$-demethylase in both diabetic male and female rat liver cytosolic fractions [35]. A recent study confirmed that there was an increase in the activity of $N$-demethylase and UDP-glucuronosyltransferase (UGT) in male rats treated with the methanolic, aqueous, and total alkaloid extract from M. speciosa leaves [36].

There have been no reports that M. speciosa extract and MG alter CES activity, but this enzyme can be affected by several other chemicals. For example, dexamethasone and phenobarbital have been shown to induce CES in humans [37]. Organophosphate and carbamate insecticides have caused an enzyme inhibition through phosphorylation or carbamylation of the serine residue at the active center of the enzyme [15,38]. The only study that has identified metabolites of MG in rat and human urine postulated its metabolic pathways in both species [17]. MG was metabolized by hydrolysis of the methylesterat position 16 and $O$-demethylation of the 9-methoxy group and of the 17-methoxy group, followed by oxidation of the intermediate aldehydes to carboxylic acids or reduction to alcohols and a combination of some steps. No information about the enzymes responsible for the metabolism of MG has been reported. However, the substrate specificity of CES1 has been documented [39]. CES1 preferentially catalyses the hydrolysis of compounds esterified with a small alcohol group. That corresponds to the structure of MG and is consistent with hydrolysis of the methylester at position 16. It may be possible that MG binds to CES1 and is a substrate for hydrolysis. In addition, the results of the study suggest that MG causes inhibition of CES1, and thus inhibits the metabolism of PM. It would be possible and helpful to further investigate and confirm the interaction of MG with CES1, by determination of CES1 activity and possibly in appropriate in vitro studies.

The metabolic ratio in plasma and/or urine has been a valuable tool for enzyme activity evaluation [40]. Without pretreatment of MG and AE, PMRs increased with time because metabolite levels increased while PM levels decreased. After pretreatment, PMRs decreased and the extent of those decreases increased with time. This might be a result of the decrease of metabolite (PBAlc) levels and the increase of PM levels. As noted, there was a relative increase in trans-PM levels and a significant decrease in PBAlc levels during the terminal phase of PM elimination. The elimination kinetics shows that after pretreatment, PM was cleared more slowly while PBAlc was eliminated more rapidly. That is probably due to inhibition of PM hydrolysis catalyzed by CES and further metabolism 
of PBAlc to aldehyde and acid metabolites. Since the levels of PBAld and PBAcid were not measured, whether MG and AE induce activities of CYPs, ADH, and ALDH involving in conversion of PBAlc to PBAld and PBAcid is unclear.

Effects of single and multiple doses of either MG or AE administration on the PMR were similar. With repeated doses, the drug will accumulate in the body and reach a steady state until dosing stops. If the dosing interval is less than four half-lives, accumulation will occur. The accumulation is indexed by the accumulation factor, which equals one divided by the fraction lost in one dosing interval. When the drug is given once every half-life, the accumulation factor is 2 . This means that the concentration after intermittent doses at steady state will be twice that at the same time after the first dose. A previous study [18] has reported an elimination half-life of $9.4 \mathrm{~h}$ after an oral dose of MG in rats. In this work, MG and AE were administered once a day, which is about 2.6 times its elimination half-life. The accumulation factor is 1.31. It is likely that the steady state levels after four doses were only slightly higher than after a single dose. Therefore, no major additional inhibitory effect can occur as compared to a single dose.

The decrease in PMR due to MG and AE in the present study was different as seen from the smaller extent of reduction in the PMR caused by AE compared with MG. With regard to the procedure for preparation of $\mathrm{AE}$ and $\mathrm{MG}$ [18,20,21], the yield of $\mathrm{MG}$ was reported to be $1.27 \mathrm{~g}$ per $2.5 \mathrm{~g}$ of $\mathrm{AE}$, i.e., $50.8 \%$. The amount of MG in the AE preparation was 1.27 times higher, compared to that in the $\mathrm{MG}$ preparation. Hence, the different effects may be due to different active constituents in the preparations. MG was 98\% pure [18], whereas AE contained the main indole alkaloid (MG) and other minor constituents such as speciogynine, speciociliatine, paynantheine, 7-hydroxymitragynine, including newly discovered compounds, like 7 $\beta$-hydroxy-7H-mitraciliatine and isospeciofoleine [41]. Some of these minor substances may counteract the inhibitory effect of MG.

In the present study, we have described the elimination kinetic parameters in male Wistar rats following a single dose $(460 \mathrm{mg} / \mathrm{kg})$ of PM. The values of $\mathrm{k}_{\mathrm{el}}$ and $t_{1 / 2}$ el without pretreatment for all groups were considered the baseline. The kel $\left(0.059-0.062 \mathrm{~h}^{-1}\right)$ and $t_{1 / 2}$ el $(11.19-11.71 \mathrm{~h})$ obtained in this study were comparable to those from previous work [42]. The authors investigated the toxicokinetics of PM in male Sprague-Dawley rats and reported the rate constants for the terminal elimination phase $(\beta)$ and the half-life at the $\beta$ phase of $0.056 \mathrm{~h}^{-1}$ and $12.37 \mathrm{~h}$, respectively, after an oral dose of $460 \mathrm{mg} / \mathrm{kg}$ of PM.

The toxic effects of PM appeared in the present study. The dose of PM (460 mg/kg) was below the acute oral $\mathrm{LD}_{50}$ (median lethal dose) value in the adult rats, i.e., $1200-1500 \mathrm{mg} / \mathrm{kg}$ [14,43], and was not lethal to the animals. Following an oral administration of PM, the animals (without pretreatment) showed the typical symptoms for type-I pyrethroids toxicity such as hypersensitivity and tremors. These symptoms were exaggerated in the group that concurrently received either MG or AE and PM (data not shown). This may be due to the decrease in the rate of hydrolysis, which is important for the rate of degradation, and elimination of trans-PM. Plasma cis-PM was not determined in this work due to the lack of a pure standard. However, since hydrolysis of cis-PM is slower than that for the transisomer, and this isomer has a significantly higher affinity for the sodium channel than the transisomer, this may also contribute to the higher toxicity of PM [15]. 


\section{Conclusions}

MG and other alkaloids derived from M. speciosa exhibited toxicokinetic interactions with PM in rats. The interaction seems to be due to inhibition of PM hydrolysis presumably caused by CES, delaying PM elimination and enhancing PBAlc elimination. The findings suggest that humans consuming a kratom cocktail might be at higher risk of developing neurotoxicity from PM.

\section{Acknowledgments}

The authors acknowledge the financial support from Prince of Songkla University for the year 2010 (Project code SCI530214S), the Graduate School, the Natural Product Research Center, and the Department of Pharmacology, Faculty of Science. We are grateful to Brian Hodgson for assistance with English writing.

\section{Author Contributions}

Benjamas Janchawee and Sathaporn Prutipanlai conceived and designed the experiments; Kachamas Srichana, Benjamas Janchawee, Sathaporn Prutipanlai and Pritsana Raungrut performed the experimental work. Kachamas Srichana, Benjamas Janchawee and Sathaporn Prutipanlai analyzed the data; Niwat Keawpradub contributed the alkaloid MG. Benjamas Janchawee wrote and revised the manuscript.

\section{Conflicts of Interest}

The authors declare no conflict of interest.

\section{References}

1. Shellard, E.J.; Houghton, P.J.; Reasha, M. The Mitragyna speciosa of Asia. Part XXXI. The alkaloids of Mitragyna speciosa Korth from Thailand. Planta Med. 1978, 27, 26-36.

2. Jansen, K.L.; Prast, C.J. Psychoactive properties of mitragynine (kratom). J. Psychoact. Drugs 1988, 20, 455-457.

3. Maurer, H.H. Chemistry, pharmacology, and metabolism of emerging drugs of abuse. Ther. Drug Monit. 2010, 32, 544-549.

4. Saingam, D.; Assanangkornchai, S.; Geater, A.F.; Balthip, Q. Pattern and consequences of krathom (Mitragyna speciosa Korth.) use among male villagers in southern Thailand: A qualitative study. Int. J. Drug Policy 2013, 24, 351-358.

5. Prozialeck, W.C.; Jivan, J.K.; Andurkar, S.V. Pharmacology of Kratom: An emerging botanical agent with stimulant, analgesic and opioid-like effects. J. Am. Osteopath Assoc. 2012, 112, 792-799.

6. Takayama, H. Chemistry and pharmacology of analgesic indole alkaloids from the rubiaceous plant, Mitragyna speciosa. Chem. Pharm. Bull. 2004, 52, 916-928.

7. Chittrakarn, S.; Sawangjaroen, K.; Prasettho, S.; Janchawee, B.; Keawpradub, N. Inhibitory effects of kratom leaf extract (Mitragyna speciosa Korth.) on the rat gastrointestinal tract. J. Ethnopharmacol. 2008, 116, 173-178. 
8. Mossadeq, W.M.S.; Sulaiman, M.R.; Mohamad, T.A.T.; Chiong, H.S.; Zakaria, Z.A.; Jabit, M.L.; Baharuldin, M.T.H.; Israf, D.A. Anti-inflammatory and antinociceptive effects of Mitragyna speciosa Korth methanolic extract. Med. Princ. Pract. 2009, 18, 378-384.

9. Tsuchiya, S.; Miyashita, S.; Yamamoto, M.; Horie, S.; Sakai, S.I.; Aimi, N.; Takayama, H.; Watanabe, K. Effect of mitragynine, derived from Thai folk medicine, on gastric acid secretion through opioid receptor in anesthetized rats. Eur. J. Pharmacol. 2002, 443, 185-188.

10. Purintrapiban, J.; Keawpradub, N.; Kansenalak, S.; Chittrakarn, S.; Janchawee, B.; Sawangjaroen, K. Study on glucose transport in muscle cells by extracts from Mitragyna speciosa (Korth) and mitragynine. Nat. Prod. Res. 2011, 25, 1379-1387.

11. Boyer, E.W.; Babu, K.M.; Adkins, J.E.; McCurdy, C.R.; Halpern, J.H. Self-treatment of opioid withdrawal using kratom (Mitragynia speciosa korth). Addiction 2008, 103, 1048-1050.

12. Tungtananuwat, W.; Lawanprasert, S. Fatal 4x100; home-made kratom juice cocktail. J. Health Res. 2010, 24, 43-47.

13. Shafer, T.J.; Meyer, D.A.; Crofton, K.M. Developmental neurotoxicity of pyrethroid insecticides: Critical review and future research needs. Environ. Health Perspect. 2005, 113, 123 136.

14. Soderlund, D.M.; Clark, J.M.; Sheets, L.P.; Mullin, L.S.; Piccirillo, V.J.; Sargent, D.; Stevens, J.T.; Weiner, M.L. Mechanisms of pyrethroid neurotoxicity: Implications for cumulative risk assessment. Toxicology 2002, 171, 3-59.

15. Sogorb, M.A.; Vilanova, E. Enzymes involved in the detoxification of organophosphorus, carbamate and pyrethroid insecticides through hydrolysis. Toxicol. Lett. 2002, 128, 215-228.

16. Nakamura, Y.; Sugihara, K.; Sone, T.; Isobe, M.; Ohta, S.; Kitamura, S. The in vitro metabolism of a pyrethroid insecticide, permethrin, and its hydrolysis products in rats. Toxicology 2007, 235, 176184.

17. Philipp, A.A.; Wissenbach, D.K.; Zoerntlein, S.W.; Klein, O.N.; Kanogsunthornrat, J.; Maurer, H.H. Studies on the metabolism of mitragynine, the main alkaloid of the herbal drug Kratom, in rat and human urine using liquid chromatography-linear ion trap mass spectrometry. J. Mass Spectrom. 2009, 44, 1249-1261.

18. Janchawee, B.; Keawpradub, N.; Chittrakarn, S.; Prasettho, S.; Wararatananurak, P.; Sawangjareon, K. A high-performance liquid chromatographic method for determination of mitragynine in serum and its application to a pharmacokinetic study in rats. Biomed. Chromatogr. 2007, 21, 176-183.

19. Botpiboon, A. Effects of caffene and codeine on pharmacokinetics and antinociceptive activity of alkaloid extract from leaves of kratom (Mitragyna speciosa Korth.). M.Sc. Thesis, Prince of Songkla University, Songkhla, 2009.

20. Houghton, P.J.; Latiff, A.; Said, I.M. Alkaloids from Mitragyna speciosa. Phytochemistry 1991, 30, 347-350.

21. Ponglux, D.; Wongseripipatana, S.; Takayama, H.; Kikuchi, M.; Kurihara, M.; Kitajima, M.; Aimi, N.; Sakai, S. A New Indole Alkaloid, 7 alpha-Hydroxy-7H-mitragynine, from Mitragyna speciosa in Thailand. Planta Med. 1994, 60, 580-581.

22. Council for International Organizations of Medical Sciences. Available online: http://www.cioms. ch/images/stories/CIOMS/IGP2012.pdf (accessed on 25 October 2014). 
23. National Centre for the Replacement Refinement \& Reduction of Animals in Research. Available online: http://www.nc3rs.org.uk/sites/default/files/documents/Guidelines/NC3Rs\%20ARRIVE\% 20Guidelines\%202013.pdf (accessed on 25 October 2014).

24. Junting, L.; Chuichang, F. Solid phase extraction method for rapid isolation and clean-up of some synthetic pyrethroid insecticides from human urine and plasma. Forensic Sci. Int. 1991, 51, 89-93.

25. Abu-Qare, A.W.; Abou-Donia, M.B. Simultaneous determination of malathion, permethrin, DEET (N,N-diethyl-m-toluamide), and their metabolites in rat plasma and urine using high performance liquid chromatography. J. Pharm. Biomed. Anal. 2001, 26, 291-299.

26. U.S. Food and Drug Administration. Available online: http://www.fda.gov/downloads/Drugs /Guidances/ucm070107.pdf (accessed on 1 November 2014).

27. Choi, J.; Rose, R.L.; Hodgson, E. In vitro human metabolism of permethrin: The role of human alcohol and aldehyde dehydrogenases. Pestic. Biochem. Phys. 2002, 74, 117-128.

28. Satoh, T.; Hosokawa, M. Structure, function and regulation of carboxylesterases. Chem.-Biol. Interact. 2006, 162, 195-211.

29. Scollon, E.J.; Starr, J.M.; Godin, S.J.; DeVito, M.J.; Hughes, M.F. In vitro metabolism of pyrethroid pesticides by rat and human hepatic microsomes and cytochrome P450 isoforms. Drug Metab. Dispos. 2009, 37, 221-228.

30. Hanapi, N.A.; Azizi, J.; Ismail, S.; Mansor, S.M. Evaluation of selected Malaysian medicinal plants on phase I drug metabolizing enzymes, CYP2C9, CYP2D6 and CYP3A4 activities in vitro. Int. J. Pharmacol. 2010, 6, 494-499.

31. Hanapi, N.A.; Ismail, S.; Mansor, S.M. Inhibitory effect of mitragynine on human cytochrome P450 enzyme activities. Pharmacogn. Res. 2013, 5, 241-246.

32. Kong, W.M.; Chik, Z.; Ramachandra, M.; Subramaniam, U.; Raja Aziddin, R.E.; Mohamed, Z. Evaluation of the effects of mitragyna speciosa alkaloid extract on cytochrome P450 enzymes using a high throughput assay. Molecules 2011, 16, 7344-7356.

33. Lim, E.L.; Seah, T.C.; Koe, X.F.; Wahab, H.A.; Adenan, M.I.; Jamil, M.F.A.; Majid, M.I.A.; Tan, M.L. In vitro evaluation of cytochrome P450 induction and the inhibition potential of mitragynine, a stimulant alkaloid. Toxicol. in Vitro 2013, 27, 812-824.

34. Azizi, J.; Ismail, S.; Mordi, M.N.; Ramanathan, S.; Said, M.I.M.; Mansor, S.M. In vitro and in vivo effects of three different mitragyna speciosa korth leaf extracts on phase II drug metabolizing enzymes-glutathione transferases (GSTs). Molecules 2010, 15, 432-441.

35. Anwar, R.; Ismail, S.; Mansor, S.M. In vitro effect of mitragynine on activity of drug metabolizing enzymes, n-demethylase and glutathione s-transferase in streptozotocin-induced diabetic rats. Pharmacologyonline 2012, 1, 68-75.

36. Azizi, J.; Ismail, S.; Mansor, S.M.Mitragyna speciosa Korth leaves extracts induced the CYP450 catalyzed aminopyrine-N-demethylase (APND) and UDP-glucuronosyltransferase (UGT) activities in male Sprague-Dawley rat livers. Drug Metabol. Drug Interact. 2013, 28, 95-105.

37. Zhu, W.; Song, L.; Zhang, H.; Matoney, L.; Lecluyse, E.; Yan, B. Dexamethasone differentially regulates expression of carboxylesterase genes in humans and rats. Drug Metab. Dispos. 2000, 28, 186-191.

38. Hodgson, E.; Rose, R.L. Metabolic interactions of agrochemicals in humans. Pest. Manag. Sci. 2008, 64, 617-621. 
39. Imai, T. Human carboxylesterase isozymes: Catalytic properties and rational drug design. Drug Metab. Pharmacokinet. 2006, 21, 173-185.

40. Arnaud, M.J. Pharmacokinetics and metabolism of natural methylxanthines in animal and man. In Methylxanthines; Springer Berlin Heidelberg: Berlin, Germany, 2011; pp 33-91.

41. Ali, Z.; Demiray, H.; Khan, I.A. Isolation, characterization, and NMR spectroscopic data of indole and oxindole alkaloids from Mitragyna speciosa. Tetrahedron Lett. 2014, 55, 369-372.

42. Anadon, A.; Martinez-Larranaga, M.R.; Diaz, M.J.; Bringas, P. Toxicokinetics of permethrin in the rat. Toxicol. Appl. Pharmacol. 1991, 110, 1-8.

43. Cantalamessa, F. Acute toxicity of two pyrethroids, permethrin, and cypermethrin in neonatal and adult rats. Arch. Toxicol. 1993, 67, 510-513.

(C) 2015 by the authors; licensee MDPI, Basel, Switzerland. This article is an open access article distributed under the terms and conditions of the Creative Commons Attribution license (http://creativecommons.org/licenses/by/4.0/). 